\title{
Electrochemiluminescence Based on a Dual Carbon Ultramicroelectrode with the Confined Steady-state Annihilation
}

Minghan Wang, ${ }^{\dagger}$ Junjie Liu, ${ }^{\dagger}$ Xu Liang, ${ }^{\dagger}$ Rongyao Gao, ${ }^{\ddagger}$ Yiming Zhou, ${ }^{\ddagger}$ Xin Nie, ${ }^{\dagger}$ Yi

Shao, ${ }^{\dagger}$ Yan Guan, ${ }^{\dagger}$ Limin Fu, ${ }^{\ddagger}$ Jianping Zhang ${ }^{\ddagger}$ and Yuanhua Shao*†

† Beijing National Laboratory for Molecular Sciences, College of Chemistry and Molecular Engineering, Peking University, Beijing 100871, China

${ }^{\ddagger}$ Department of Chemistry, Renmin University of China, Beijing 100872, China

\section{Table of contents}

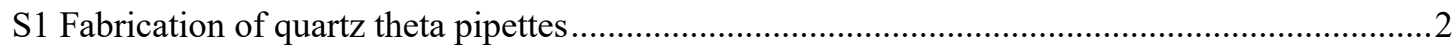

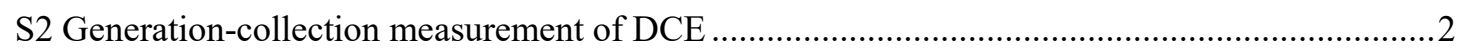

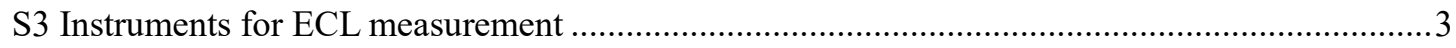

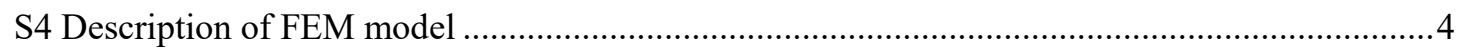

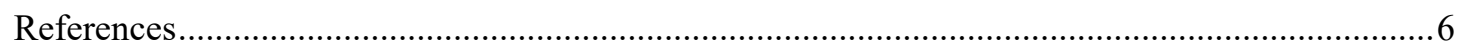

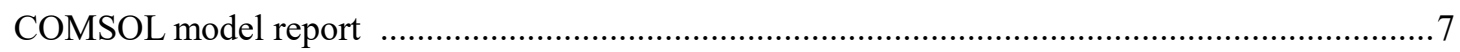

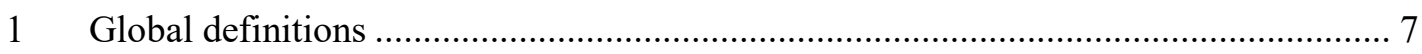

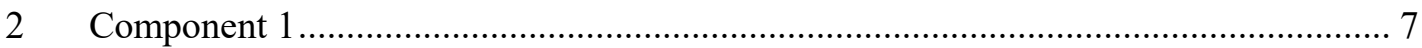

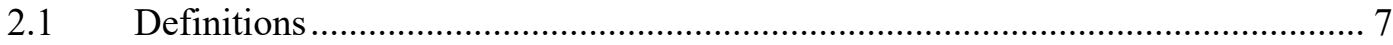

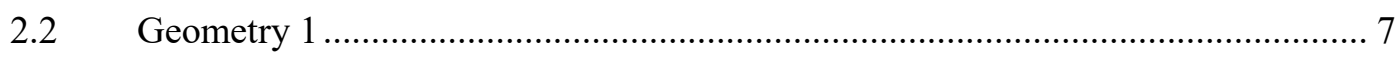

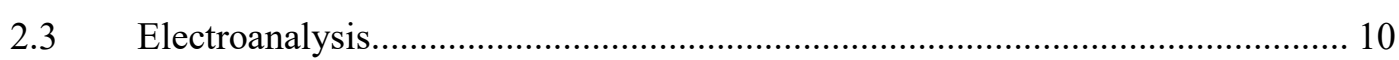

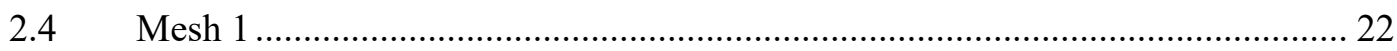

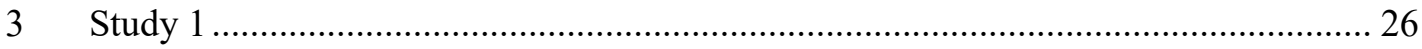

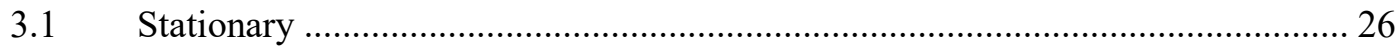

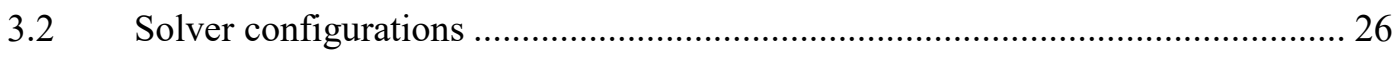




\section{S1 Fabrication of Quartz Theta Pipettes}

A two-line program was used to prepare the scaffold of the submicrometer-sized dual carbon electrodes in this work in order to keep stability of the pulling. The program is listed below in Table S1.

Table S1 Paraments for the preparation of theta quartz pipette in submicron scale.

\begin{tabular}{lllll}
\hline Heat & Filament & Velocity & Delay & Pull \\
\hline 770 & 3 & 20 & 128 & 50 \\
\hline 660 & 2 & 15 & 132 & 150 \\
\hline
\end{tabular}

\section{S2 Generation/collection Measurement of the DCE}

The prepared and characterized DCE was then measured in the generation/collection mode, with the $\mathrm{Ru}\left(\mathrm{NH}_{3}\right)_{6}{ }^{3+/ 2+}$ redox couple. The potential of the generation electrode was swept from $0 \mathrm{~V}$ to $0.45 \mathrm{~V}$ for the reduction of $\mathrm{Ru}\left(\mathrm{NH}_{3}\right)_{6}{ }^{3+}$ to $\mathrm{Ru}\left(\mathrm{NH}_{3}\right)_{6}{ }^{2+}$, while the potential of the collection electrode was held constant at $0 \mathrm{~V}$ for the oxidation of $\mathrm{Ru}\left(\mathrm{NH}_{3}\right)_{6}{ }^{2+}$ back to $\mathrm{Ru}\left(\mathrm{NH}_{3}\right)_{6}{ }^{3+}$. The linear sweep voltammograms of each barrel of dual carbon electrode in $5 \mathrm{mM} \mathrm{Ru}\left(\mathrm{NH}_{3}\right)_{6} \mathrm{Cl}_{3}$ aqueous solution were shown in Figure S1, and a reasonable collection efficiency of $31.6 \%$ was obtained for the measured DCE. This result is similar to or much larger than the generation-collection efficiencies of other probe-type dual electrode devices in the previous work (ca. 30\% for dual carbon electrodes $^{\mathrm{S} 1}$ and $<10 \%$ for dual submicrometer-sized platinum electrode $\mathrm{S}^{\mathrm{S} 2}$ ).

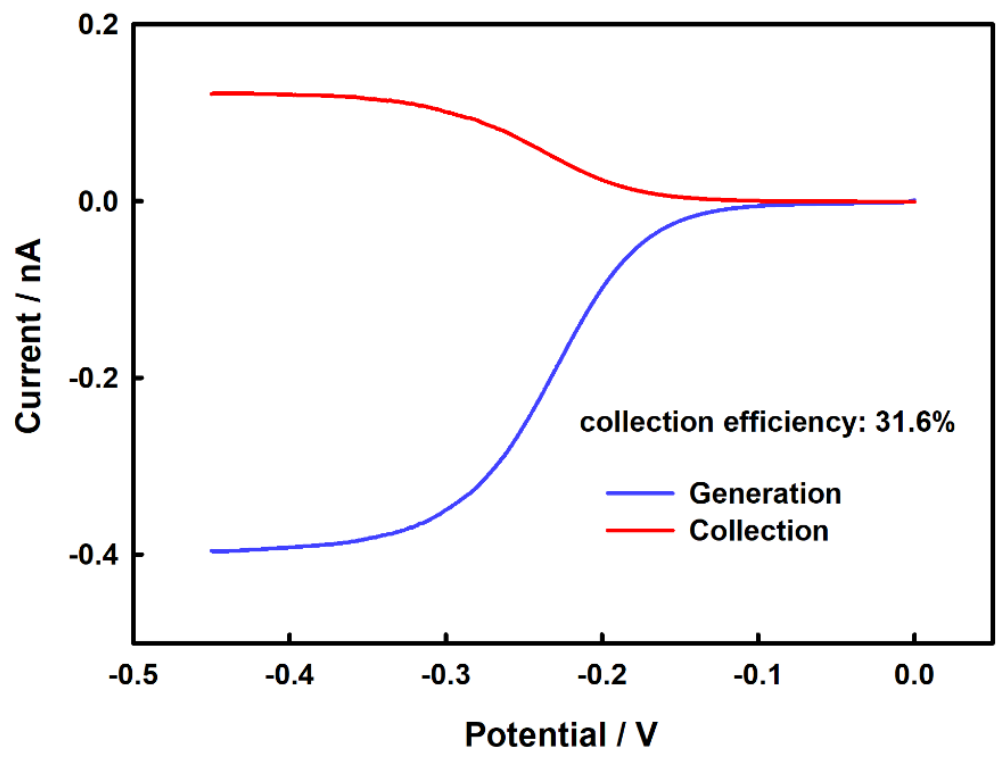

Figure S1. LSV for generation (blue line) and corresponding collection current (red line) of DCE in $5 \mathrm{mM} \mathrm{Ru}\left(\mathrm{NH}_{3}\right)_{6} \mathrm{Cl}_{3}$ aqueous solution containing $0.1 \mathrm{M} \mathrm{KCl}$, scan rate: $10 \mathrm{mV} / \mathrm{s}$. 


\section{S3 Instruments for ECL Measurements}

As discussed in the main text, the setup for ECL measurement consists of an electrochemical workstation and a microscopy platform. The detection scheme for ECL microscopy and the figure of homemade quartz cell were shown in Figures S2 and S3, respectively.

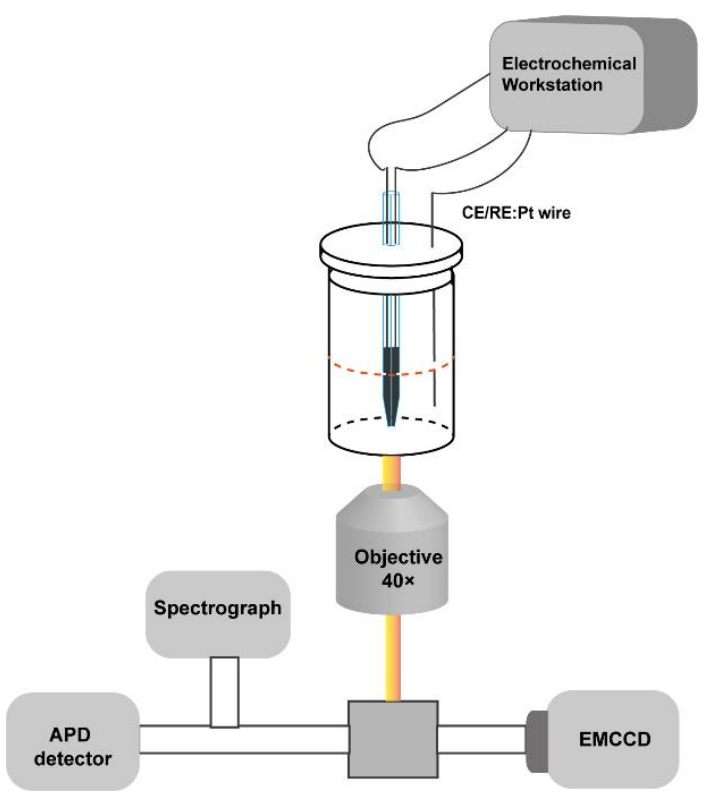

Figure S2. Schematic diagram of microscopy platform for ECL measurements

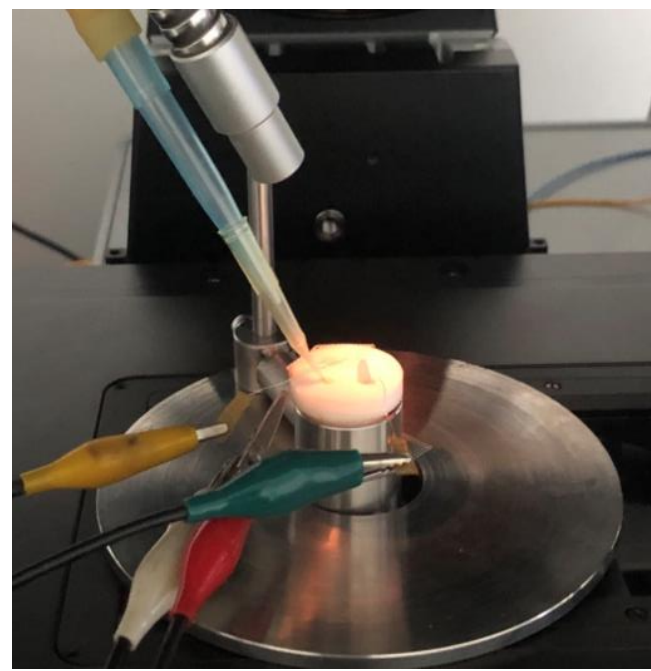

Figure S3. The homemade quartz cell for ECL measurement 


\section{S4 Description of FEM model}

A two-dimensional FEM model of DCE was constructed on the basis of its geometry observed by SEM, which consists of the two planar electrodes (semi-axis $=410 \mathrm{~nm}$ ) and an insulating septum $(d=60 \mathrm{~nm})$ between the electrodes. A rectangular around the tip of DCE was also added to describe the reactions and fluxes in the bulk solution.

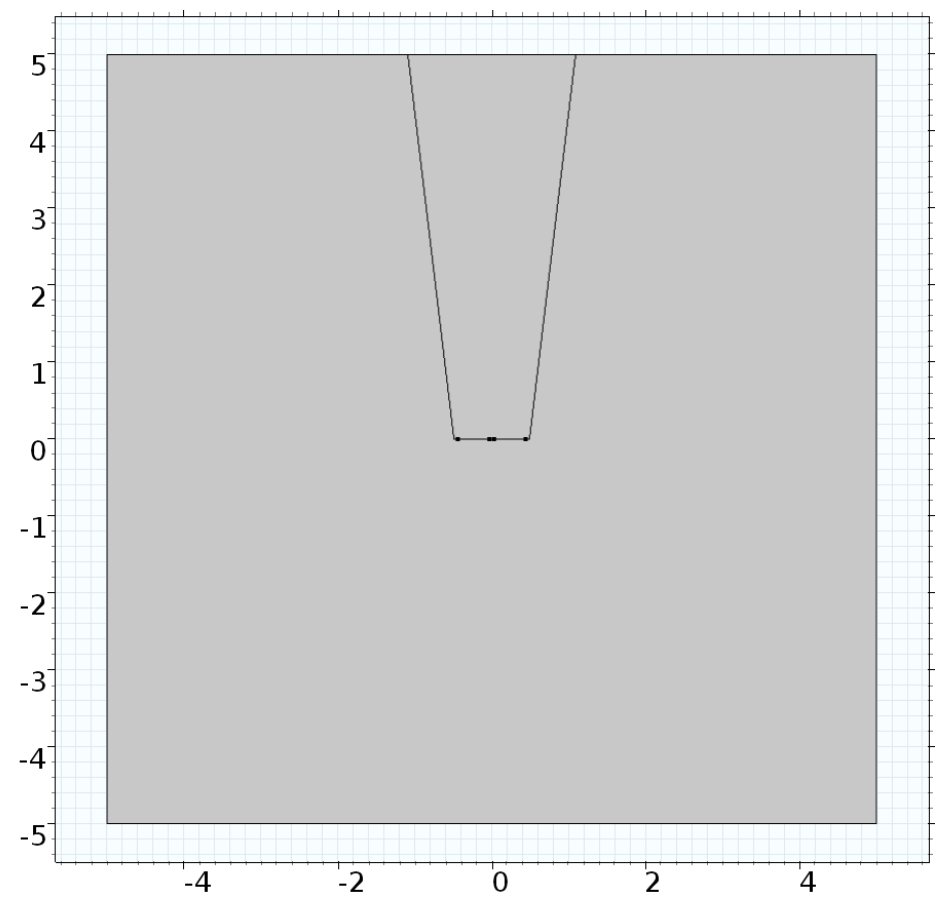

Figure S4. Geometric model of dual carbon electrode

Herein, the concentrations of $\left[\mathrm{Ru}(\mathrm{bpy})_{3}\right]^{+},\left[\mathrm{Ru}(\mathrm{bpy})_{3}\right]^{2+},\left[\mathrm{Ru}(\mathrm{bpy})_{3}\right]^{3+}$ and $\left[\mathrm{Ru}(\mathrm{bpy})_{3}\right]^{2+*}$ were denoted by $c_{1}, c_{2}, c_{3}$ and $c_{4}$, respectively.

We treated the diffusion of all species in solution purely on mass transport mechanism (no convection and electrical migration), which was proved to follow the Fick's second laws:

$$
\frac{\partial c_{i}}{\partial t}=D \nabla^{2} c_{i}
$$

We assumed that the diffusion coefficients $(D)$ of four $\left[R u(b p y)_{3}\right]$ species are equal, for $10^{-9} \mathrm{~m}^{2}$. $s^{-1} . \mathrm{S} 3$

The reaction mechanism of annihilation ECL is:

$$
\begin{gathered}
{\left[R u(b p y)_{3}\right]^{2+}+e \rightarrow \quad\left[R u(b p y)_{3}\right]^{+}} \\
{\left[R u(b p y)_{3}\right]^{2+}-e \rightarrow \quad\left[R u(b p y)_{3}\right]^{3+}} \\
{\left[R u(b p y)_{3}\right]^{+}+\left[R u(b p y)_{3}\right]^{3+} \stackrel{k_{\text {ann }}}{\longrightarrow}\left[R u(b p y)_{3}\right]^{2+}+\left[R u(b p y)_{3}\right]^{2+*}} \\
{\left[R u(b p y)_{3}\right]^{2+*} \stackrel{k_{4}}{\rightarrow}\left[R u(b p y)_{3}\right]^{2+}+h v}
\end{gathered}
$$

We implemented the reduction of $\left[\mathrm{Ru}(\mathrm{bpy})_{3}\right]^{2+}$ to $\left[\mathrm{Ru}(\mathrm{bpy})_{3}\right]^{+}$(eq S2) occurred at the right barrel of DCE, while the oxidation of $\left[\mathrm{Ru}(\mathrm{bpy})_{3}\right]^{2+}$ to $\left[\mathrm{Ru}(\mathrm{bpy})_{3}\right]^{3+}$ (eq S3) occurred at the left barrel. 
At the electrode surface, we considered the reaction kinetics on each electrode follows ButlerVolmer formalism:

$$
i=n F k_{0}\left(c_{\text {red }}\left(\frac{(1-\alpha) F \eta}{R T}\right)-c_{o x} \exp \left(\frac{-\alpha F \eta}{R T}\right)\right)
$$

where the $F, R$, and $T$ represent the Faraday constant, gas constant and temperature, respectively. Here we set $\alpha=0.5$ as the charge transfer coefficient, and $k_{0}=0.01 \mathrm{~m} \cdot \mathrm{s}^{-1}$ as the reaction rate. And the overpotential of each electrode was calculated as following: $\eta_{a}=\varphi_{a}-\varphi_{e q, a}=1.2 \mathrm{~V}-$ $0.91 \mathrm{~V}=0.29 \mathrm{~V}, \eta_{c}=\varphi_{c}-\varphi_{e q, c}=-1.8 \mathrm{~V}-(-1.61 \mathrm{~V})=-0.19 \mathrm{~V}$.

The annihilation reaction (eq4) and (eq5) mainly occurred in the solution near the electrode tip, which follows the homogeneous second-order kinetics. ${ }^{S 4}$ Considering the generation and consumption of the four species, the reaction rates $R_{\mathrm{i}=1,2,3,4}$ were calculated as following:

$$
\begin{gathered}
R_{1}=-k_{a n n} c_{1} c_{3} \\
R_{2}=k_{a n n} c_{1} c_{3}+k_{4} c_{4} \\
R_{3}=-k_{a n n} c_{1} c_{3} \\
R_{4}=k_{a n n} c_{1} c_{3}-k_{4} c_{4}
\end{gathered}
$$

We set a fast annihilation reaction rate of $k_{a n n}=10^{10} M^{-1} s^{-1}$, and the lifetime of $\left[R u(b p y)_{3}\right]^{2+*}$ of $1 \mu s\left(k_{4}=10^{6} s^{-1}\right)$ according to the previous report. ${ }^{\mathrm{S} 5}$

As for the Generation-collection mode, the reaction pathway of DCE is:

$$
\begin{aligned}
& {\left[R u(b p y)_{3}\right]^{2+}+e \rightarrow \quad\left[R u(b p y)_{3}\right]^{+}} \\
& {\left[R u(b p y)_{3}\right]^{+}-e \rightarrow \quad\left[R u(b p y)_{3}\right]^{2+}}
\end{aligned}
$$

We considered that the reduction of $\left[\mathrm{Ru}(\mathrm{bpy})_{3}\right]^{2+}$ (eq.11) occurred at the right barrel, whereas the regeneration of $\left[\mathrm{Ru}(\mathrm{bpy})_{3}\right]^{2+}$ (eq.S12) occurred at the left barrel. In GC mode, we only need to take into account the standard potential $\varphi_{e q, c}=-1.61$, and the potential of the left electrode was set as $0 \mathrm{~V}$ for electroanalysis. The simulated concentration profiles of $\left[\mathrm{Ru}(\mathrm{bpy})_{3}\right]^{+}$and $\left[\mathrm{Ru}(\mathrm{bpy})_{3}\right]^{2+}$ were shown in Figure S5.
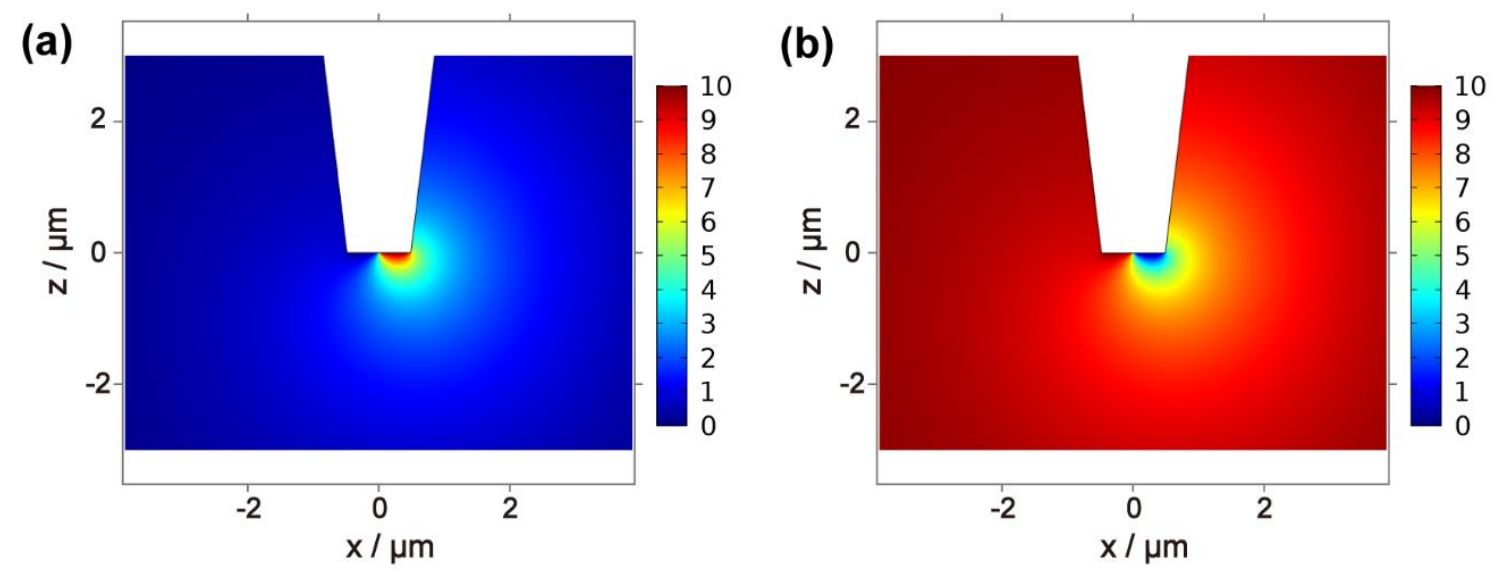

Figure S5. Finite Element Simulation of concentration profiles for (a) $\left[R u(b p y)_{3}\right]^{+}$and (b) $\left[\mathrm{Ru}(\mathrm{bpy})_{3}\right]^{2+}$ in GC mode. 
In order to better visualize the concentration profiles of $\mathrm{Ru}$ species around the tip of electrode, a 3-dimentional FEM geometric model of DCE was also constructed, and the slices (x-z plane) of simulated results were shown in Figure S6. The result could be used as supplemental material for Figure 7 in the main text.
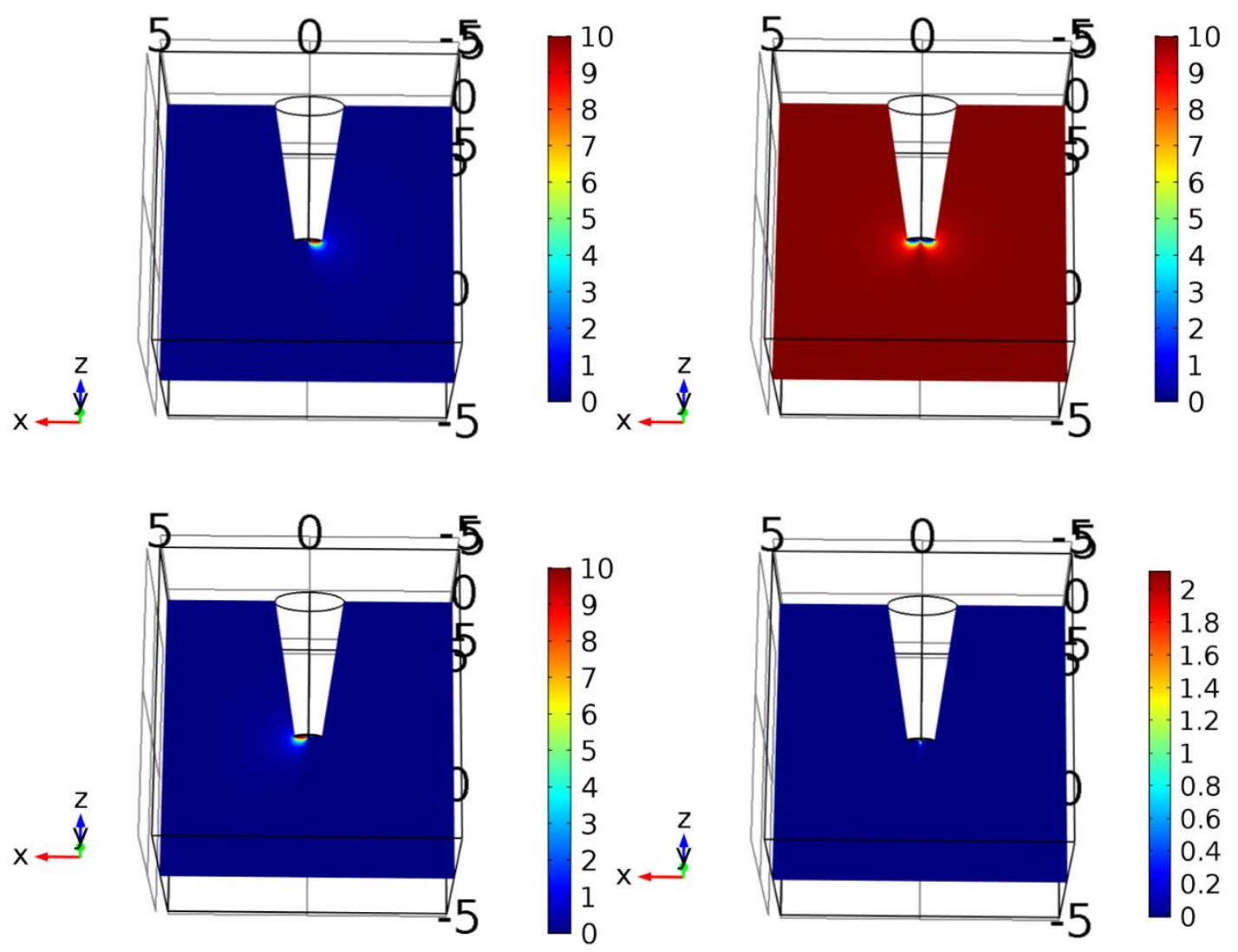

Figure S6. Finite Element Simulation of concentration profiles for (a) $\left[\mathrm{Ru}(\mathrm{bpy})_{3}\right]^{+}$, (b) $\left[\mathrm{Ru}(\mathrm{bpy})_{3}\right]^{2+}$, (c) $\left[\mathrm{Ru}(\mathrm{bpy})_{3}\right]^{3+}$ and (d) $\left[\mathrm{Ru}(\mathrm{bpy})_{3}\right]^{2+*}$ in the solution near DCE under steady state. Diffusion coefficient for all species: $D=10^{-9} \mathrm{~m}^{2} / \mathrm{s}$, concentration of the parent molecule in bulk solution: $c_{\mathrm{A}}=10$ $\mathrm{mM}$.

\section{References}

S1 McKelvey, K.; Nadappuram, B. P.; Actis, P.; Takahashi, Y.; Korchev, Y. E.; Matsue, T.; Robinson, C.; Unwin, P. R. Anal. Chem. 2013, 85, 7519-7526.

S2 Yang, C.; Sun, P. Anal. Chem. 2009, 81, 7496-7500.

S3 Amatore, C.; Fosset, B.; Karolyn, M. M.; Wightman, R. M. Anal. Chem. 1993, 65, 2311-2316.

S4 Rodriguez-Lopez, J.; Shen, M.; Nepomnyashchii, A. B.; Bard, A. J. J. Am. Chem. Soc. 2012, 134, 9240-9250.

S5 Al-Kutubi, H.; Voci, S.; Rassaei, L.; Sojic, N.; Mathwig, K. Chem. Sci. 2018, 9, 8946-8950. 


\section{COMSOL Model Report}

\section{GLOBAL DEFINITIONS}

\begin{tabular}{|l|l|} 
Date & Aug 11, 2020 1:47:42 PM \\
\hline
\end{tabular}

Global settings

\begin{tabular}{|l|l|}
\hline Name & DCE model.mph \\
\hline Path & C:IUsers \WMH\Desktop\Dual|DCE model.mph \\
\hline COMSOL version & COMSOL 5.2 (Build: 166) \\
\hline
\end{tabular}

Used products

COMSOL Multiphysics

Batteries \& Fuel Cells Module

\section{COMPONENT 1}

\subsection{DEFINITIONS}

\subsubsection{Coordinate Systems}

\section{Boundary System 1}

\begin{tabular}{|l|l|}
\hline Coordinate system type & Boundary system \\
\hline Tag & sys1 \\
\hline
\end{tabular}

\subsection{GEOMETRY 1}

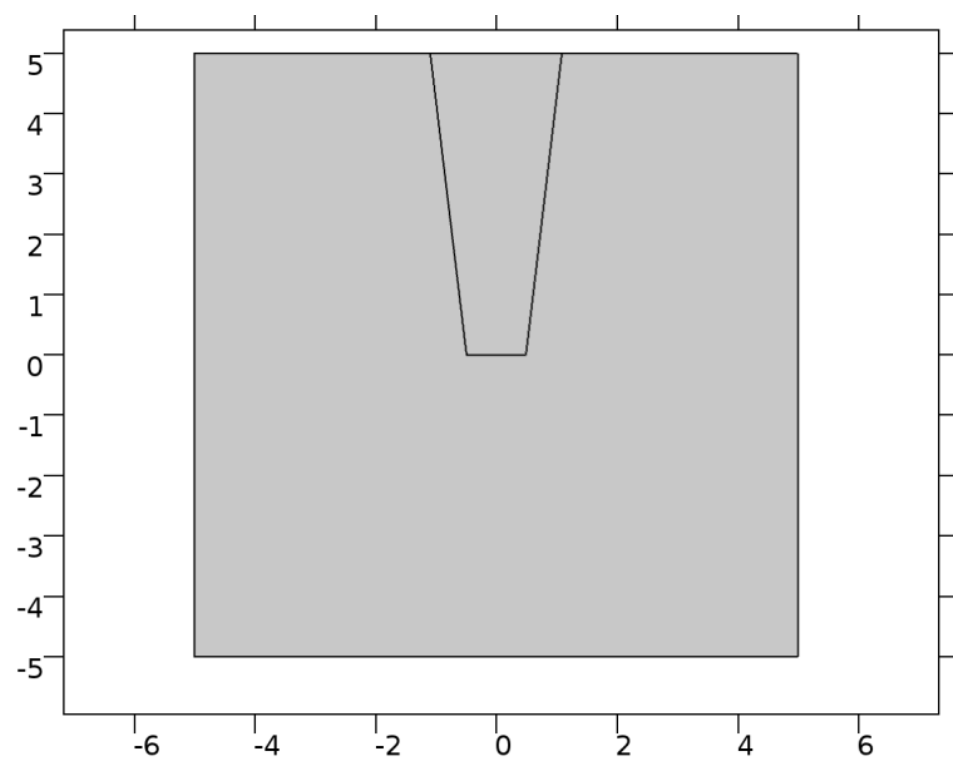

Geometry 1 
Units

\begin{tabular}{|l|l|}
\hline Length unit & $\mu \mathrm{m}$ \\
\hline Angular unit & $\mathrm{rad}$ \\
\hline
\end{tabular}

Geometry statistics

\begin{tabular}{|l|l|}
\hline Description & Value \\
\hline Space dimension & 2 \\
\hline Number of domains & 2 \\
\hline Number of boundaries & 15 \\
\hline Number of vertices & 14 \\
\hline
\end{tabular}

\subsubsection{Rectangle 1 (r1)}

\section{Position}

\begin{tabular}{|l|l|}
\hline Description & Value \\
\hline Position & $\{-0.49,0\}$ \\
\hline
\end{tabular}

Size

\begin{tabular}{|l|l|}
\hline Description & Value \\
\hline Width & 0.98 \\
\hline Height & 5 \\
\hline
\end{tabular}

\subsubsection{Point 1 (pt1)}

\section{Point}

\begin{tabular}{|l|l|}
\hline Description & Value \\
\hline Point coordinate & $\{-0.03,0\}$ \\
\hline
\end{tabular}

\subsubsection{Point 2 (pt2)}

Point

\begin{tabular}{|l|l|}
\hline Description & Value \\
\hline Point coordinate & $\{0.03,0\}$ \\
\hline
\end{tabular}

\subsubsection{Point 3 (pt3)}

\section{Point}

\begin{tabular}{|l|l|}
\hline Description & Value \\
\hline Point coordinate & $\{-0.44,0\}$ \\
\hline
\end{tabular}


2.2.5 Point 4 (pt4)

Point

\begin{tabular}{|l|l|}
\hline Description & Value \\
\hline Point coordinate & $\{0.44,0\}$ \\
\hline
\end{tabular}

\subsubsection{Rectangle 2 (r2)}

\section{Position}

\begin{tabular}{|l|l|}
\hline Description & Value \\
\hline Position & $\{0,0\}$ \\
\hline
\end{tabular}

Size

\begin{tabular}{|l|l|}
\hline Description & Value \\
\hline Width & 10 \\
\hline Height & 10 \\
\hline
\end{tabular}

\subsubsection{Polygon 1 (pol1)}

Object type

\begin{tabular}{|l|l|}
\hline Description & Value \\
\hline Type & Solid \\
\hline
\end{tabular}

Coordinates

\begin{tabular}{|l|l|}
\hline Description & Value \\
\hline Data source & Table \\
\hline
\end{tabular}

Coordinates

\begin{tabular}{|l|l|}
\hline $\mathbf{x}(\boldsymbol{\mu m})$ & $\mathbf{y}(\boldsymbol{\mu m})$ \\
\hline-0.49 & 0 \\
\hline-1.09 & 5 \\
\hline-0.49 & 5 \\
\hline
\end{tabular}

2.2.8 Polygon 2 (pol2)

Object type

\begin{tabular}{|l|l|}
\hline Description & Value \\
\hline Type & Solid \\
\hline
\end{tabular}

Coordinates 


\begin{tabular}{|l|l|}
\hline Description & Value \\
\hline Data source & Table \\
\hline
\end{tabular}

\section{Coordinates}

\begin{tabular}{|l|l|}
\hline $\mathbf{x}(\boldsymbol{\mu m})$ & $\mathbf{y}(\boldsymbol{\mu m})$ \\
\hline 0.49 & 0 \\
\hline 1.09 & 5 \\
\hline 0.49 & 5 \\
\hline
\end{tabular}

\subsubsection{Union 1 (uni1)}

\section{Compose}

\begin{tabular}{|l|l|}
\hline Description & Value \\
\hline Keep interior boundaries & Off \\
\hline
\end{tabular}

\subsection{ELECTROANALYSIS}

\section{Used products}

COMSOL Multiphysics

Batteries \& Fuel Cells Module

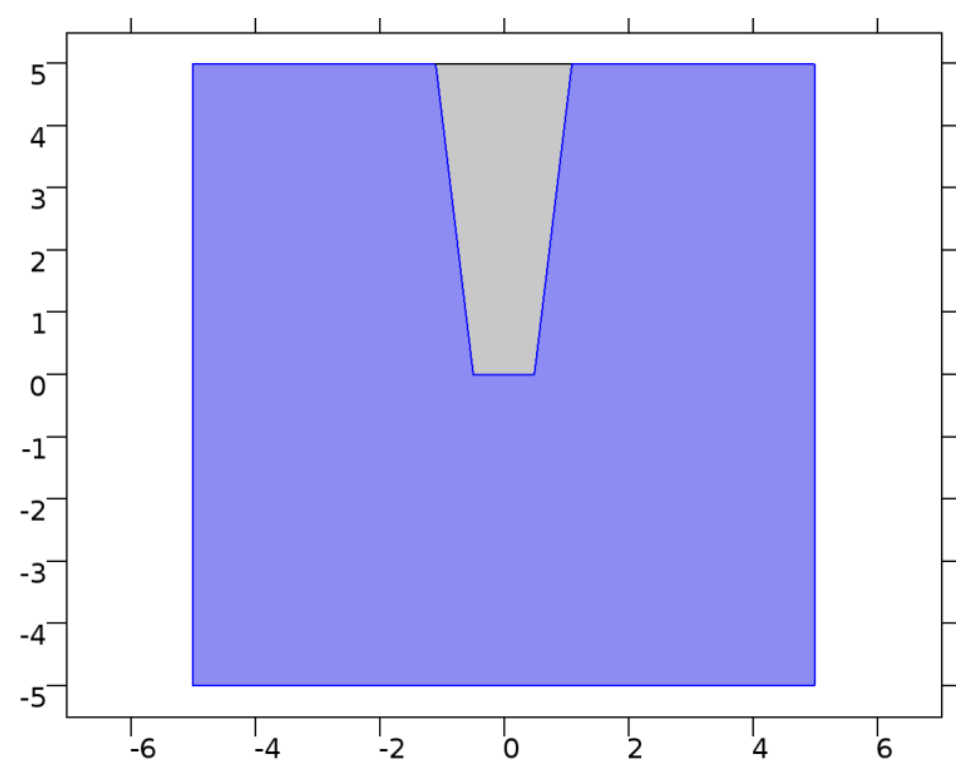

Electroanalysis

\section{Selection}

\begin{tabular}{|l|l|}
\hline Geometric entity level & Domain \\
\hline Selection & Domain 1 \\
\hline
\end{tabular}




\section{Equations}

$$
\begin{aligned}
& \nabla \cdot\left(-D_{i} \nabla c_{i}\right)=R_{i} \\
& \mathbf{N}_{i}=-D_{i} \nabla c_{i} \\
& \phi_{1}=0
\end{aligned}
$$

\section{Settings}

\begin{tabular}{|l|l|}
\hline Description & Value \\
\hline Concentration & Linear \\
\hline Compute boundary fluxes & On \\
\hline Apply smoothing to boundary fluxes & On \\
\hline Value type when using splitting of complex variables & Real \\
\hline Convection & Off \\
\hline Migration in electric field & Off \\
\hline Thickness & $1[\mathrm{~m}]$ \\
\hline Streamline diffusion & On \\
\hline Crosswind diffusion & On \\
\hline Equation residual & Approximate residual \\
\hline Crosswind diffusion type & Do Carmo and Galeão \\
\hline Convective term & Non - conservative form \\
\hline
\end{tabular}

\subsubsection{Transport Properties 1}

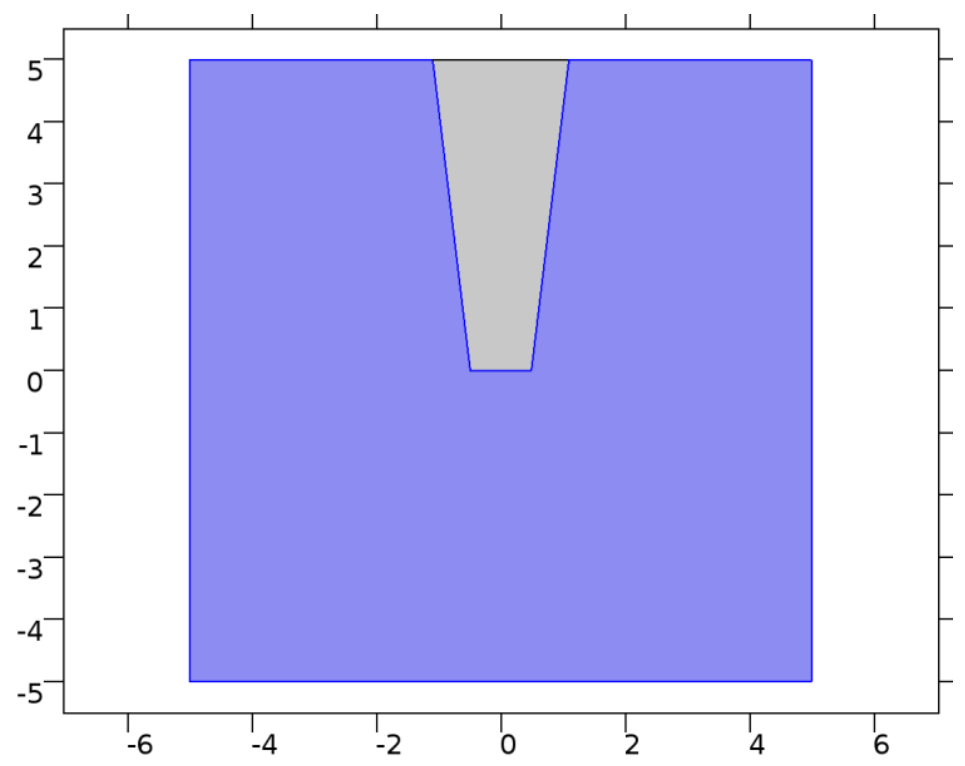

Transport Properties 1 


\section{Selection}

\begin{tabular}{|l|l|}
\hline Geometric entity level & Domain \\
\hline Selection & Domain 1 \\
\hline
\end{tabular}

\section{Equations}

$$
\begin{aligned}
& \nabla \cdot\left(-D_{i} \nabla c_{i}\right)=R_{i} \\
& \mathbf{N}_{i}=-D_{i} \nabla c_{i}
\end{aligned}
$$

\section{Settings}

\begin{tabular}{|l|l|}
\hline Description & Value \\
\hline Material & None \\
\hline Diffusion coefficient & User defined \\
\hline Diffusion coefficient & $\left\{\left\{1 \mathrm{e}-9\left[\mathrm{~m}^{\wedge} 2 / \mathrm{s}\right], 0,0\right\},\left\{0,1 \mathrm{e}-9\left[\mathrm{~m}^{\wedge} 2 / \mathrm{s}\right], 0\right\},\left\{0,0,1 \mathrm{e}-9\left[\mathrm{~m}^{\wedge} 2 / \mathrm{s}\right]\right\}\right\}$ \\
\hline Diffusion coefficient & User defined \\
\hline Diffusion coefficient & $\left\{\left\{1 \mathrm{e}-9\left[\mathrm{~m}^{\wedge} 2 / \mathrm{s}\right], 0,0\right\},\left\{0,1 \mathrm{e}-9\left[\mathrm{~m}^{\wedge} 2 / \mathrm{s}\right], 0\right\},\left\{0,0,1 \mathrm{e}-9\left[\mathrm{~m}^{\wedge} 2 / \mathrm{s}\right]\right\}\right\}$ \\
\hline Diffusion coefficient & User defined \\
\hline Diffusion coefficient & $\left\{\left\{1 \mathrm{e}-9\left[\mathrm{~m}^{\wedge} 2 / \mathrm{s}\right], 0,0\right\},\left\{0,1 \mathrm{e}-9\left[\mathrm{~m}^{\wedge} 2 / \mathrm{s}\right], 0\right\},\left\{0,0,1 \mathrm{e}-9\left[\mathrm{~m}^{\wedge} 2 / \mathrm{s}\right]\right\}\right\}$ \\
\hline Diffusion coefficient & User defined \\
\hline Diffusion coefficient & $\left\{\left\{1 \mathrm{e}-9\left[\mathrm{~m}^{\wedge} 2 / \mathrm{s}\right], 0,0\right\},\left\{0,1 \mathrm{e}-9\left[\mathrm{~m}^{\wedge} 2 / \mathrm{s}\right], 0\right\},\left\{0,0,1 \mathrm{e}-9\left[\mathrm{~m}^{\wedge} 2 / \mathrm{s}\right]\right\}\right\}$ \\
\hline
\end{tabular}

\subsubsection{No Flux 1}

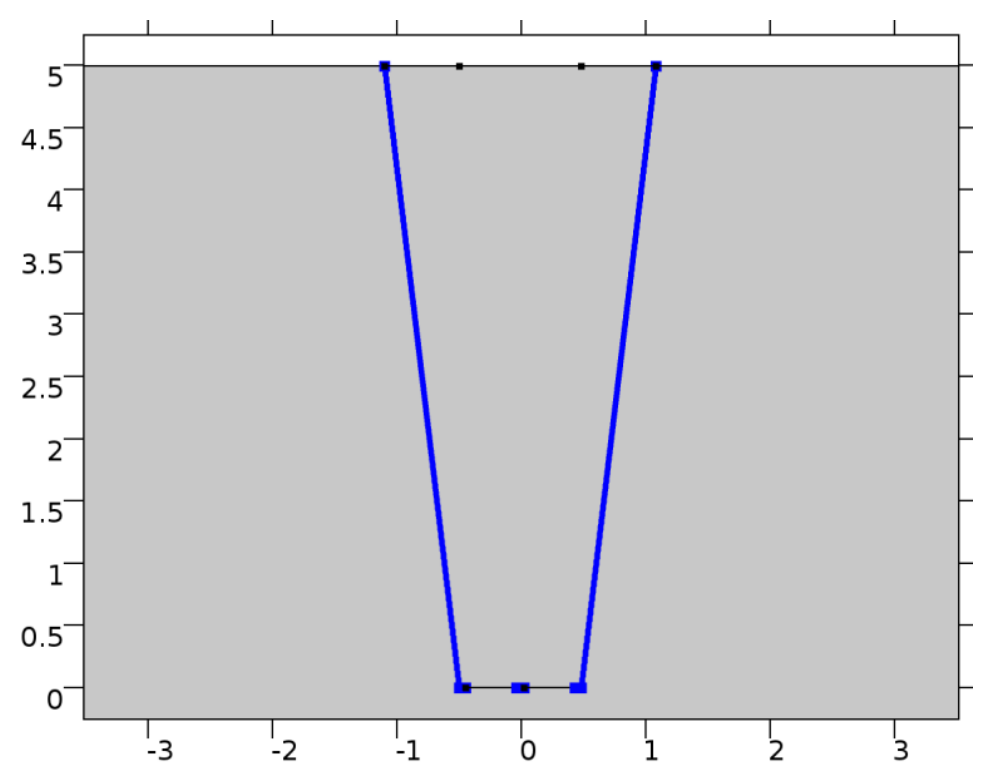

No Flux 1

\section{Selection}




\begin{tabular}{|l|l|}
\hline Geometric entity level & Boundary \\
\hline Selection & Boundaries 4, 6, 9, 11-12 \\
\hline
\end{tabular}

\section{Equations}

$$
-\mathbf{n} \cdot \mathbf{N}_{i}=0
$$

\subsubsection{Initial Values 1}

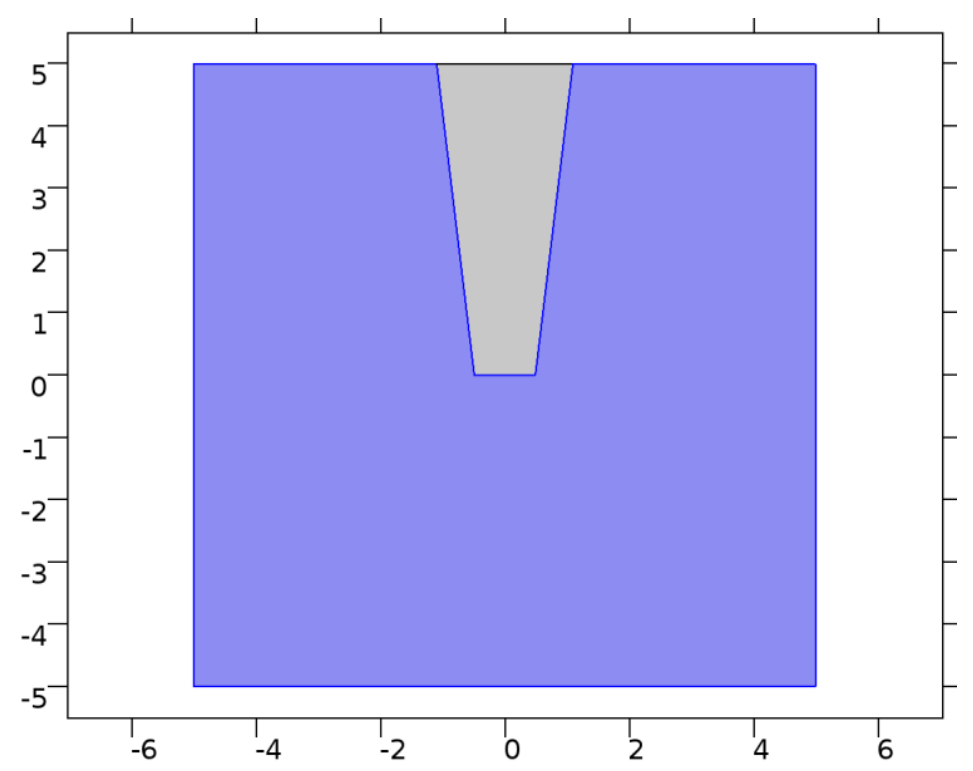

Initial Values 1

\section{Selection}

\begin{tabular}{|l|l|}
\hline Geometric entity level & Domain \\
\hline Selection & Domain 1 \\
\hline
\end{tabular}

\section{Settings}

\begin{tabular}{|l|l|}
\hline Description & Value \\
\hline Concentration & $\{0,10,0,0\}$ \\
\hline
\end{tabular}


2.3.4 Electrode Surface 1

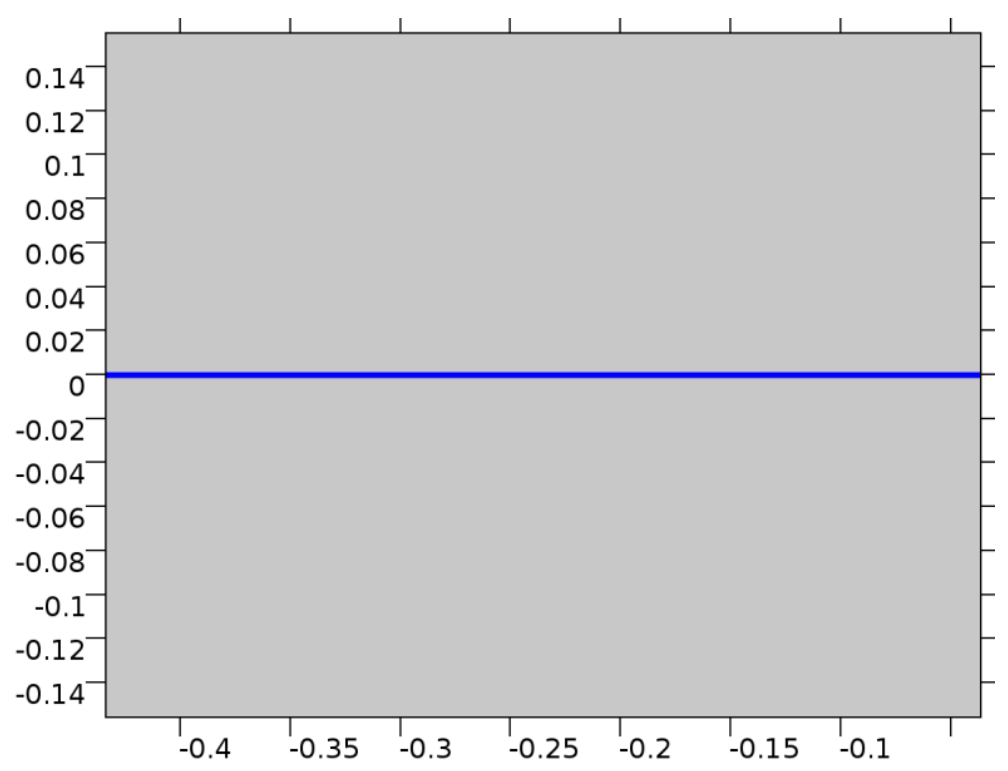

Electrode Surface 1

\section{Selection}

\begin{tabular}{|l|l|}
\hline Geometric entity level & Boundary \\
\hline Selection & Boundary 8 \\
\hline
\end{tabular}

\section{Equations}

$$
\begin{aligned}
& i_{\text {total }}=\sum_{m} i_{\text {loc, } m} \\
& -\mathbf{n} \cdot \mathbf{N}_{\mathrm{i}}=R_{i, \text { tot }}, \quad R_{i, \text { tot }}=\sum R_{i, m}
\end{aligned}
$$

\section{Settings}

\begin{tabular}{|l|l|}
\hline Description & Value \\
\hline Solve for surface concentration variables & On \\
\hline Species & \\
\hline Molar mass & 0.06355 \\
\hline Density & 8960 \\
\hline Compensate for boundary stretching & On \\
\hline Film resistance & No film resistance \\
\hline Boundary condition & Electrode potential \\
\hline Electric reference potential & User defined \\
\hline Electric reference potential & 0 \\
\hline Perturbation amplitude & 0 \\
\hline
\end{tabular}




\begin{tabular}{|l|l|}
\hline Description & Value \\
\hline Electrode potential vs reference & 1.2 \\
\hline
\end{tabular}

\section{Electrode Reaction 1}

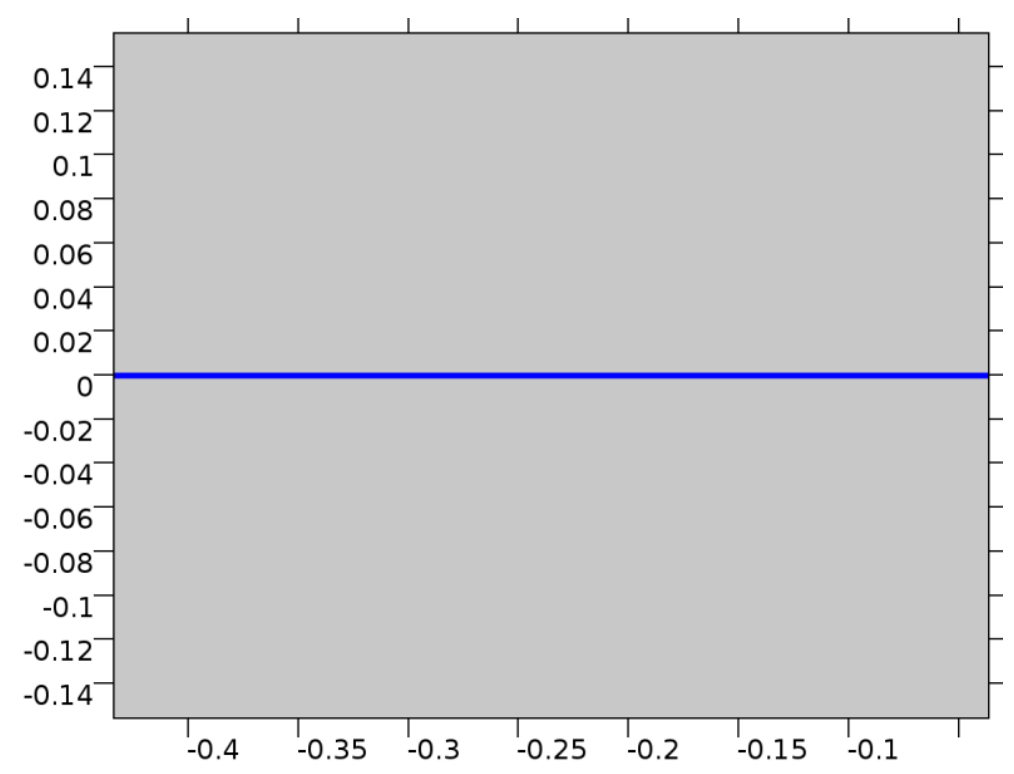

Electrode Reaction 1

\section{Selection}

\begin{tabular}{|l|l|}
\hline Geometric entity level & Boundary \\
\hline Selection & Boundary 8 \\
\hline
\end{tabular}

\section{Equations}

$$
\eta=\phi_{\mathrm{s}, \mathrm{ext}}-\phi_{1}-E_{\mathrm{eq}}
$$

\section{Settings}

\begin{tabular}{|l|l|}
\hline Description & Value \\
\hline Equilibrium potential & User defined \\
\hline Equilibrium potential & 0.91 \\
\hline Temperature derivative of equilibrium potential & User defined \\
\hline Temperature derivative of equilibrium potential & 0 \\
\hline Kinetics expression type & Electroanalytical Butler - Volmer \\
\hline Cathodic transfer coefficient & 0.5 \\
\hline Heterogenous rate constant & $0.01[\mathrm{~m} / \mathrm{s}]$ \\
\hline Number of participating electrons & 1 \\
\hline Stoichiometric coefficient & $\{0,-1,1,0\}$ \\
\hline
\end{tabular}




\subsubsection{Electrode Surface 2}

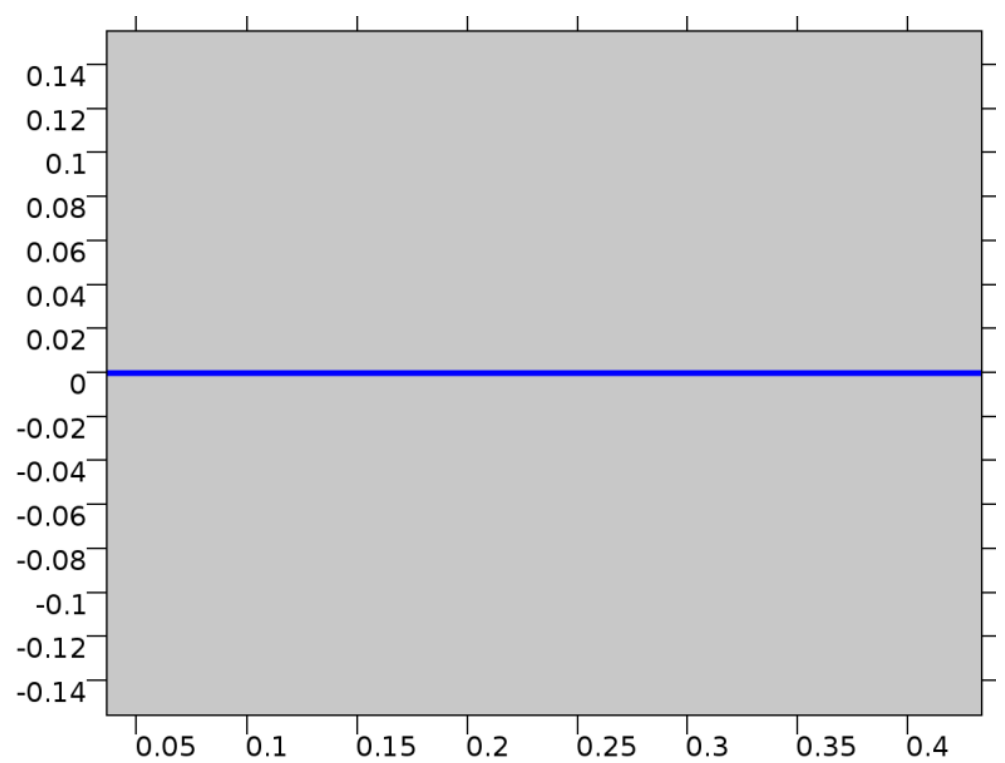

Electrode Surface 2

\section{Selection}

\begin{tabular}{|l|l|}
\hline Geometric entity level & Boundary \\
\hline Selection & Boundary 10 \\
\hline
\end{tabular}

\section{Equations}

$$
\begin{aligned}
& i_{\text {total }}=\sum_{m} i_{\text {loc }, m} \\
& -\mathbf{n} \cdot \mathbf{N}_{\mathrm{i}}=R_{i, \text { tot }}, \quad R_{i, \text { tot }}=\sum_{. m \ldots \ldots \ldots} R_{i, m}
\end{aligned}
$$

\section{Settings}

\begin{tabular}{|l|l|}
\hline Description & Value \\
\hline Solve for surface concentration variables & On \\
\hline Species & \\
\hline Molar mass & 0.06355 \\
\hline Density & 8960 \\
\hline Compensate for boundary stretching & On \\
\hline Film resistance & No film resistance \\
\hline Boundary condition & Electrode potential \\
\hline Electric reference potential & User defined \\
\hline Electric reference potential & 0 \\
\hline
\end{tabular}




\begin{tabular}{|l|l|}
\hline Description & Value \\
\hline Perturbation amplitude & 0 \\
\hline Electrode potential vs reference & -1.8 \\
\hline
\end{tabular}

\section{Electrode Reaction 1}

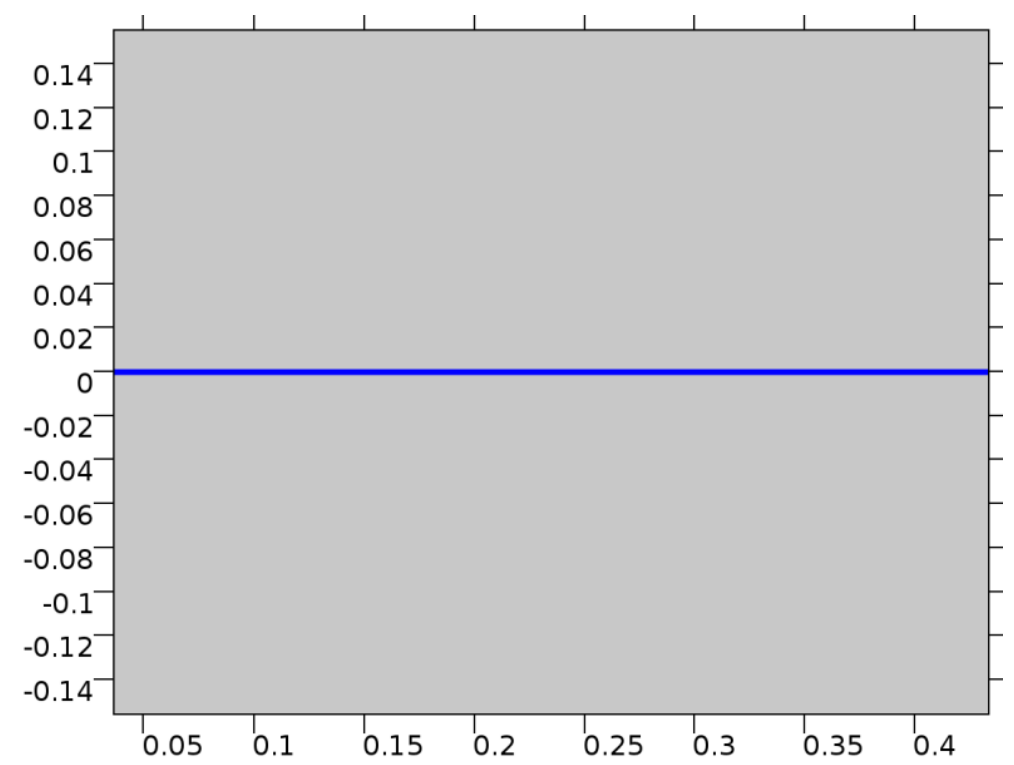

Electrode Reaction 1

\section{Selection}

\begin{tabular}{|l|l|}
\hline Geometric entity level & Boundary \\
\hline Selection & Boundary 10 \\
\hline
\end{tabular}

\section{Equations}

$$
\eta=\phi_{\mathrm{s}, \mathrm{ext}}-\phi_{1}-E_{\mathrm{eq}}
$$

\section{Settings}

\begin{tabular}{|l|l|}
\hline Description & Value \\
\hline Equilibrium potential & User defined \\
\hline Equilibrium potential & -1.72 \\
\hline Temperature derivative of equilibrium potential & User defined \\
\hline Temperature derivative of equilibrium potential & 0 \\
\hline Kinetics expression type & Electroanalytical Butler - Volmer \\
\hline Cathodic transfer coefficient & 0.5 \\
\hline Heterogenous rate constant & $0.01[\mathrm{~m} / \mathrm{s}]$ \\
\hline Number of participating electrons & 1 \\
\hline
\end{tabular}




\begin{tabular}{|l|l|}
\hline Description & Value \\
\hline Stoichiometric coefficient & $\{1,-1,0,0\}$ \\
\hline
\end{tabular}

\subsubsection{Concentration 1}

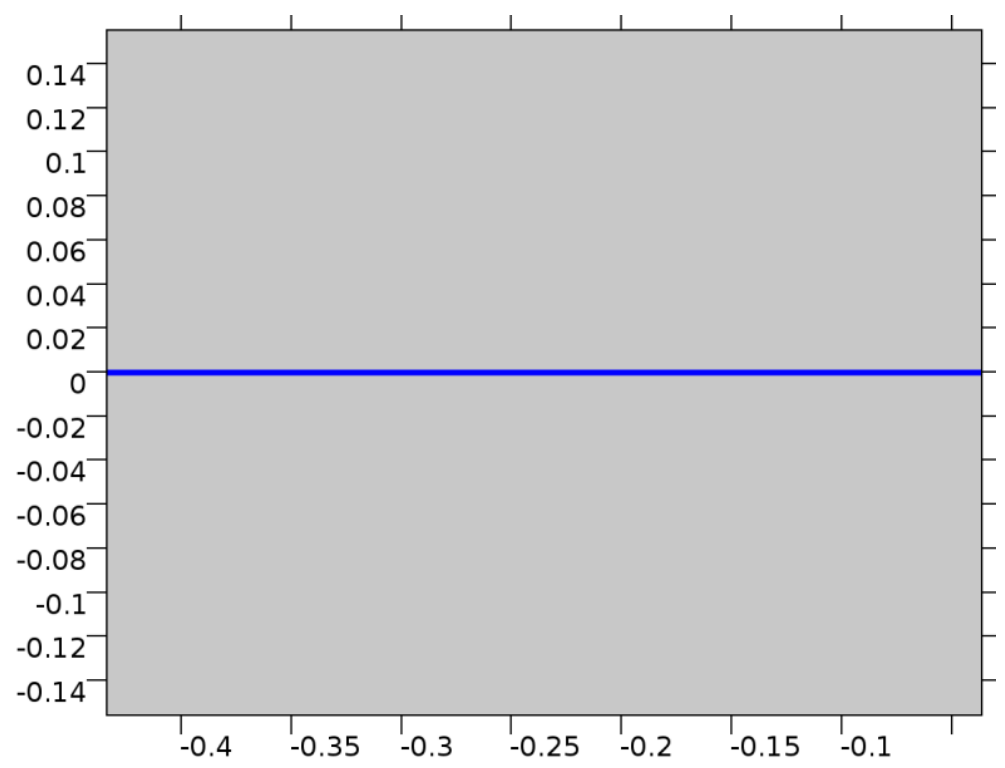

Concentration 1

\section{Selection}

\begin{tabular}{|l|l|}
\hline Geometric entity level & Boundary \\
\hline Selection & Boundary 8 \\
\hline
\end{tabular}

\section{Equations}

$$
c_{i}=c_{0, j}
$$

\section{Settings}

\begin{tabular}{|l|l|}
\hline Description & Value \\
\hline Species c1 & On \\
\hline Species c2 & On \\
\hline Species c3 & On \\
\hline Species c4 & Off \\
\hline Concentration & $\{0,0,10,0\}$ \\
\hline Apply reaction terms on & All physics (symmetric) \\
\hline Use weak constraints & Off \\
\hline Constraint method & Elemental \\
\hline
\end{tabular}


2.3.7 Concentration 2

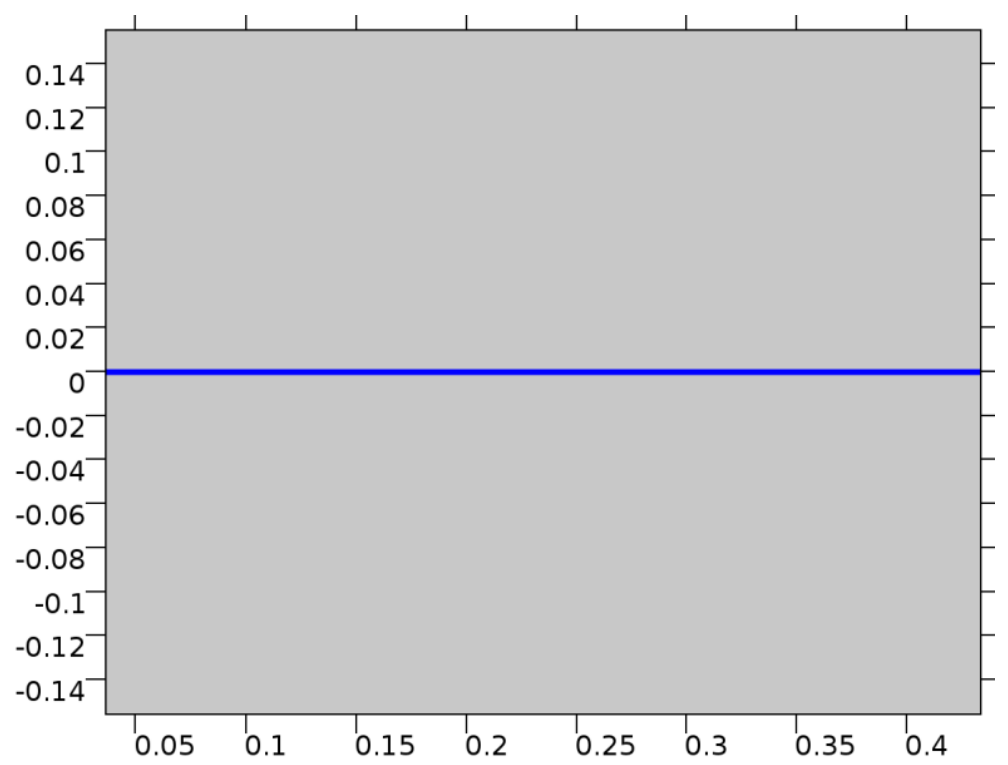

Concentration 2

\section{Selection}

\begin{tabular}{|l|l|}
\hline Geometric entity level & Boundary \\
\hline Selection & Boundary 10 \\
\hline
\end{tabular}

\section{Equations}

$$
c_{i}=c_{0, j}
$$

\section{Settings}

\begin{tabular}{|l|l|}
\hline Description & Value \\
\hline Species c1 & On \\
\hline Species c2 & On \\
\hline Species c3 & On \\
\hline Species c4 & Off \\
\hline Concentration & $\{10,0,0,0\}$ \\
\hline Apply reaction terms on & All physics (symmetric) \\
\hline Use weak constraints & Off \\
\hline Constraint method & Elemental \\
\hline
\end{tabular}




\subsubsection{Reaction 1}

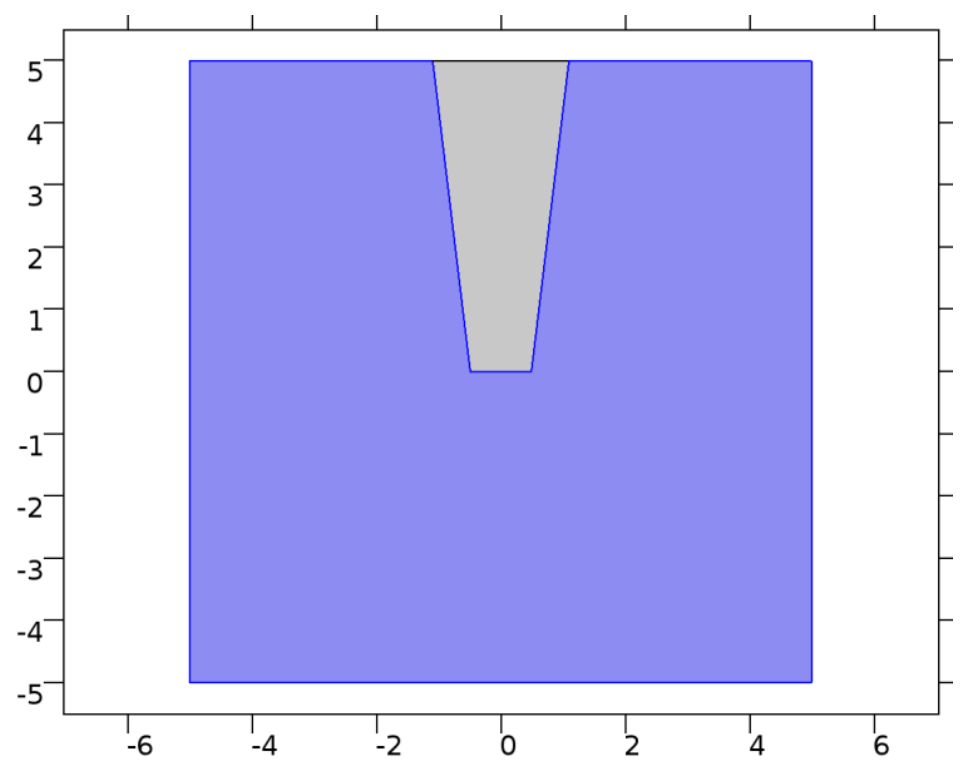

Reaction 1

\section{Selection}

\begin{tabular}{|l|l|}
\hline Geometric entity level & Domain \\
\hline Selection & Domain 1 \\
\hline
\end{tabular}

\section{Equations}

$$
\nabla \cdot\left(-D_{i} \nabla c_{i}\right)=R_{i} .
$$

\section{Settings}

\begin{tabular}{|l|l|}
\hline Description & Value \\
\hline Total rate expression & User defined \\
\hline Total rate expression & $-1 \mathrm{e} 7 *^{\mathrm{c}} 1 * \mathrm{c} 3$ \\
\hline Total rate expression & User defined \\
\hline Total rate expression & $1 \mathrm{e} 7 * \mathrm{c} 1 * \mathrm{c} 3+1 \mathrm{e} 6{ }^{*} \mathrm{c} 4$ \\
\hline Total rate expression & User defined \\
\hline Total rate expression & $-1 \mathrm{e} 7 * \mathrm{c} 1 * \mathrm{c} 3$ \\
\hline Total rate expression & $\mathrm{User}$ defined \\
\hline Total rate expression & $1 \mathrm{e} 7 * \mathrm{c} 1 * \mathrm{c} 3-1 \mathrm{e} 6 * \mathrm{c} 4$ \\
\hline
\end{tabular}


2.3.9 Concentration 3

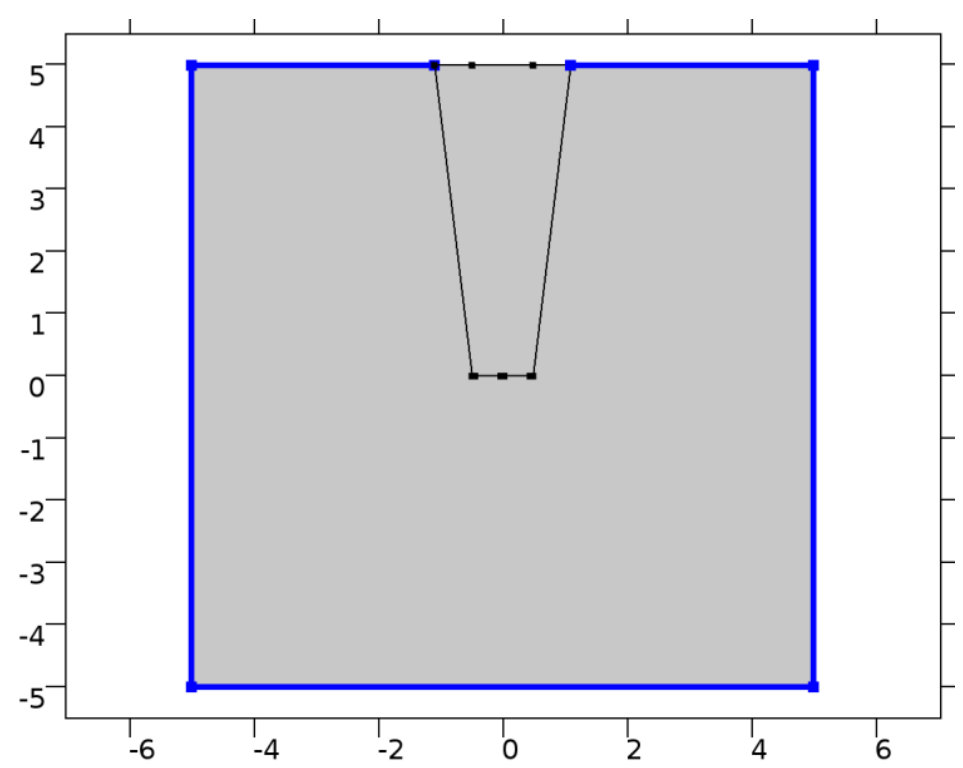

Concentration 3

\section{Selection}

\begin{tabular}{|l|l|}
\hline Geometric entity level & Boundary \\
\hline Selection & Boundaries 1-3, 14-15 \\
\hline
\end{tabular}

\section{Equations}

$$
c_{i}=c_{0, j}
$$

\section{Settings}

\begin{tabular}{|l|l|}
\hline Description & Value \\
\hline Species c1 & On \\
\hline Species c2 & On \\
\hline Species c3 & On \\
\hline Species c4 & On \\
\hline Concentration & $\{0,10,0,0\}$ \\
\hline Apply reaction terms on & All physics (symmetric) \\
\hline Use weak constraints & Off \\
\hline Constraint method & Elemental \\
\hline
\end{tabular}




\subsection{MESH 1}

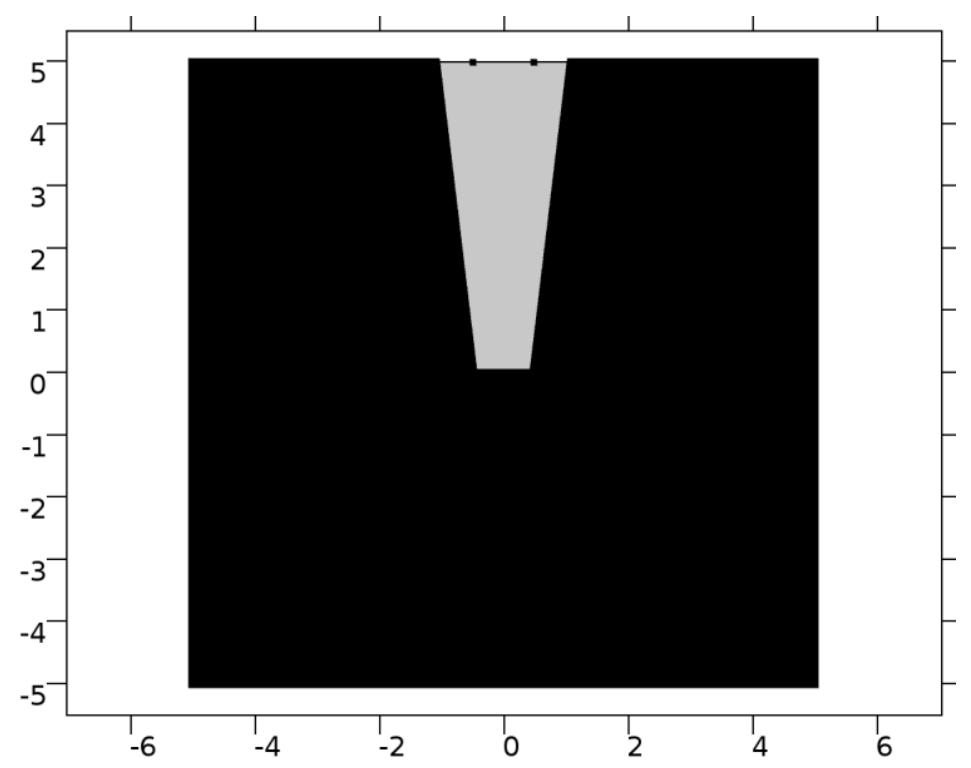

Mesh 1

\subsubsection{Size (size)}

Settings

\begin{tabular}{|l|l|}
\hline Description & Value \\
\hline Maximum element size & 0.02 \\
\hline Minimum element size & 0.002 \\
\hline Curvature factor & 0.3 \\
\hline Maximum element growth rate & 1.3 \\
\hline Custom element size & Custom \\
\hline
\end{tabular}

\subsubsection{Free Triangular 1 (ftri1)}

\section{Selection}

\begin{tabular}{|l|l|}
\hline Geometric entity level & Domain \\
\hline Selection & Domain 1 \\
\hline
\end{tabular}




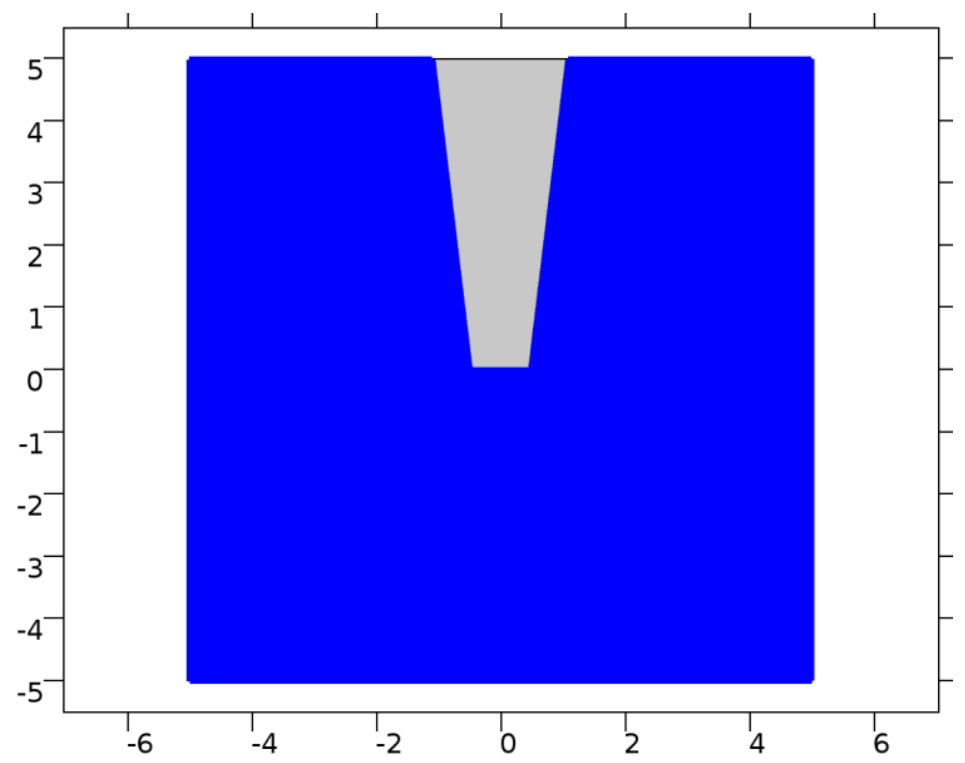

Free Triangular 1

\section{Size 1 (size1)}

\section{Selection}

\begin{tabular}{|l|l|}
\hline Geometric entity level & Boundary \\
\hline Selection & Boundary 8 \\
\hline
\end{tabular}

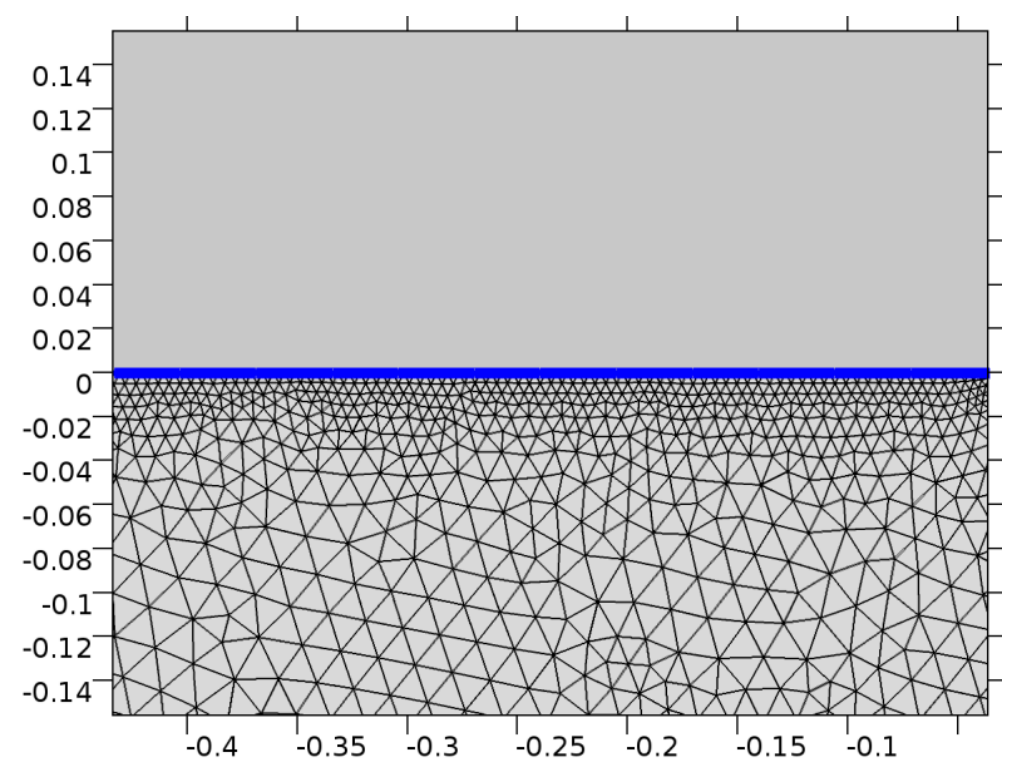

Size 1

\section{Settings}

\begin{tabular}{|l|l|}
\hline Description & Value \\
\hline Maximum element size & 0.005 \\
\hline Minimum element size & 0.00174 \\
\hline Minimum element size & Off \\
\hline
\end{tabular}




\begin{tabular}{|l|l|}
\hline Description & Value \\
\hline Curvature factor & 0.3 \\
\hline Curvature factor & Off \\
\hline Resolution of narrow regions & Off \\
\hline Maximum element growth rate & 1.3 \\
\hline Maximum element growth rate & Off \\
\hline Custom element size & Custom \\
\hline
\end{tabular}

Size 2 (size2)

\section{Selection}

\begin{tabular}{|l|l|}
\hline Geometric entity level & Boundary \\
\hline Selection & Boundary 10 \\
\hline
\end{tabular}

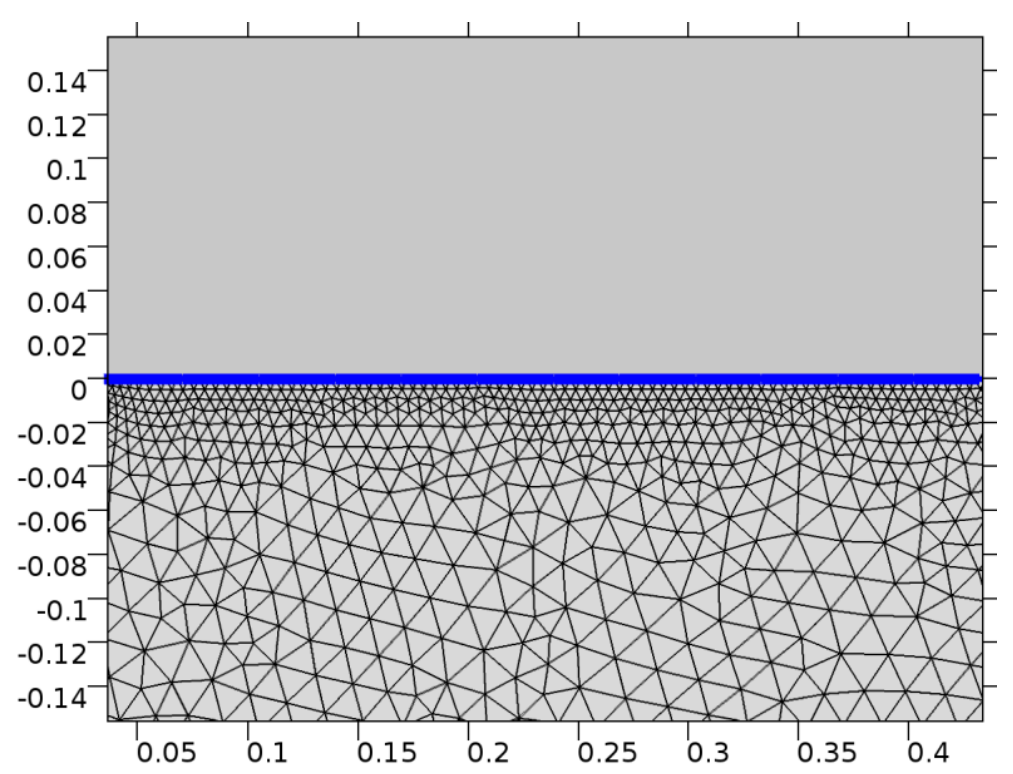

Size 2

\section{Settings}

\begin{tabular}{|l|l|}
\hline Description & Value \\
\hline Maximum element size & 0.005 \\
\hline Minimum element size & 0.00174 \\
\hline Minimum element size & Off \\
\hline Curvature factor & 0.3 \\
\hline Curvature factor & Off \\
\hline Resolution of narrow regions & Off \\
\hline
\end{tabular}




\begin{tabular}{|l|l|}
\hline Description & Value \\
\hline Maximum element growth rate & 1.3 \\
\hline Maximum element growth rate & Off \\
\hline Custom element size & Custom \\
\hline
\end{tabular}

Size 3 (size3)

\section{Selection}

\begin{tabular}{|l|l|}
\hline Geometric entity level & Boundary \\
\hline Selection & Boundary 9 \\
\hline
\end{tabular}

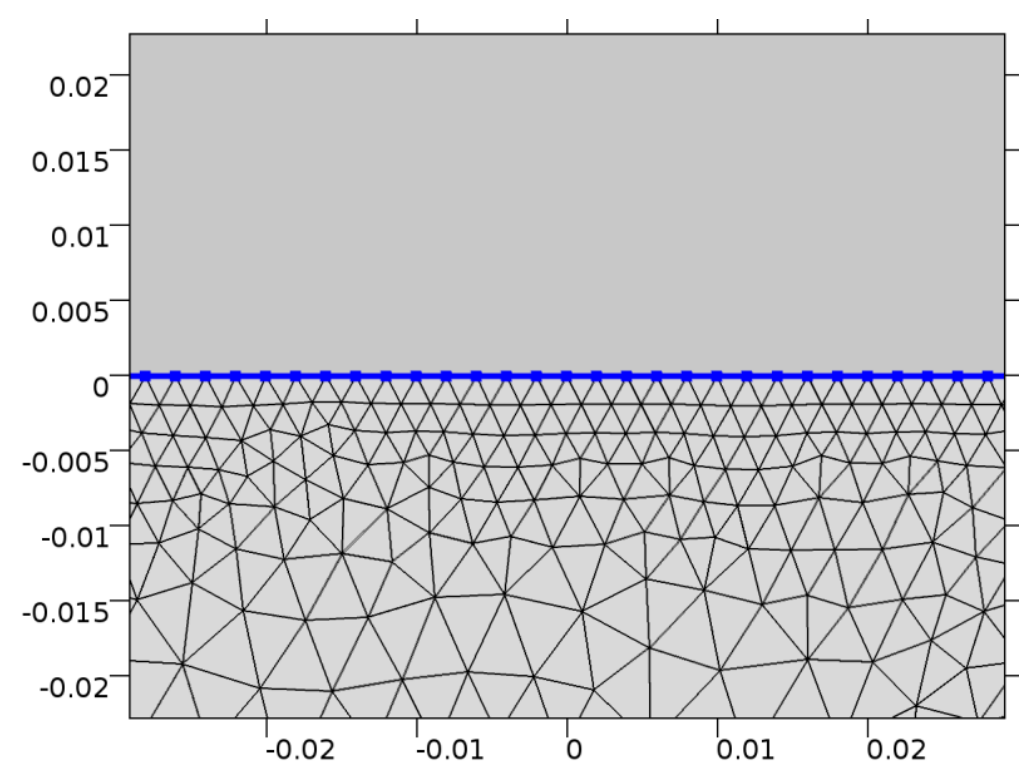

Size 3

Settings

\begin{tabular}{|l|l|}
\hline Description & Value \\
\hline Maximum element size & 0.002 \\
\hline Minimum element size & 0.00174 \\
\hline Minimum element size & Off \\
\hline Curvature factor & 0.3 \\
\hline Curvature factor & Off \\
\hline Resolution of narrow regions & Off \\
\hline Maximum element growth rate & 1.3 \\
\hline Maximum element growth rate & Off \\
\hline Custom element size & Custom \\
\hline
\end{tabular}




\section{STUDY 1}

Computation information

\begin{tabular}{|l|l|}
\hline Computation time & $5 \mathrm{~min} 36 \mathrm{~s}$ \\
\hline $\mathrm{CPU}$ & Intel(R) Core(TM) i7-10710U CPU @ 1.10GHz, 6 cores \\
\hline Operating system & Windows 8 \\
\hline
\end{tabular}

\subsection{STATIONARY}

Study settings

\begin{tabular}{|l|l|}
\hline Description & Value \\
\hline Include geometric nonlinearity & Off \\
\hline
\end{tabular}

Physics and variables selection

\begin{tabular}{|l|l|}
\hline Physics interface & Discretization \\
\hline Electroanalysis (elan) & physics \\
\hline
\end{tabular}

Mesh selection

\begin{tabular}{|l|l|}
\hline Geometry & Mesh \\
\hline Geometry1 (geom1) & mesh1 \\
\hline
\end{tabular}

\subsection{SOLVER CONFIGURATIONS}

\subsubsection{Solution 1}

Compile Equations: Stationary (st1)

Study and step

\begin{tabular}{|l|l|}
\hline Description & Value \\
\hline Use study & $\underline{\text { Study } 1}$ \\
\hline Use study step & $\underline{\text { Stationary }}$ \\
\hline
\end{tabular}

Dependent Variables 1 (v1)

\section{General}

\begin{tabular}{|l|l|}
\hline Description & Value \\
\hline Defined by study step & $\underline{\text { Stationary }}$ \\
\hline
\end{tabular}

Concentration (comp1.c1) (comp1_c1)

General

\begin{tabular}{|l|l|}
\hline Description & Value \\
\hline Field components & comp1.c1 \\
\hline
\end{tabular}


Concentration (comp1.c4) (comp1_c4)

General

\begin{tabular}{|l|l|}
\hline Description & Value \\
\hline Field components & comp1.c4 \\
\hline
\end{tabular}

Concentration (comp1.c3) (comp1_c3)

General

\begin{tabular}{|l|l|}
\hline Description & Value \\
\hline Field components & comp1.c3 \\
\hline
\end{tabular}

Concentration (comp1.c2) (comp1_c2)

General

\begin{tabular}{|l|l|}
\hline Description & Value \\
\hline Field components & comp1.c2 \\
\hline
\end{tabular}

Stationary Solver 1 (s1)

General

\begin{tabular}{|l|l|}
\hline Description & Value \\
\hline Defined by study step & Stationary \\
\hline
\end{tabular}

Fully Coupled 1 (fc1)

General

\begin{tabular}{|l|l|}
\hline Description & Value \\
\hline Linear solver & Direct 1 \\
\hline
\end{tabular}

Method and termination

\begin{tabular}{|l|l|}
\hline Description & Value \\
\hline Initial damping factor & 0.01 \\
\hline Minimum damping factor & $1.0 \mathrm{E}-6$ \\
\hline Maximum number of iterations & 50 \\
\hline
\end{tabular}

Direct 1 (d1)

\section{General}

\begin{tabular}{|l|l|}
\hline Description & Value \\
\hline Solver & PARDISO \\
\hline
\end{tabular}

